\title{
Structure multiéchelles et propriétés des matériaux du pneu
}

\author{
Marc Couty (marc.couty@fr.michelin.com) \\ Physique des Matériaux et de leurs Transformations, Centre de Technologie Michelin, 63040 Clermont-Ferrand Cedex 09
}

Derrière l'aspect uniforme

des matériaux du pneu,

se dévoile à des échelles

de quelques nanomètres

un univers complexe.

Une vision physico-chimique

du procédé de fabrication

des mélanges donne un

éclairage sur la morphologie

de répartitions aléatoires

de petits objets fractals dans

une matrice de polymère.

On discute du lien entre

les propriétés mécaniques

mesurées au niveau macro-

scopique, la structure

aux petites échelles

des matériaux et le procédé

d'obtention de cette structure.

On illustre que le couplage

entre expériences réelles et

virtuelles via la simulation

numérique est un atout fort

de la démarche scientifique.
Un pneu, c'est rond, c'est noir. Pourtant, si l'on pouvait remonter les étapes de sa fabrication, on réaliserait combien, sous cet aspect uniforme, se cache une nature intérieure beaucoup plus complexe. Chaque pneu, petit ou gros, est composé d'une vingtaine de matériaux spécifiques lui conférant ses propriétés d'élasticité, de longévité, d'adhérence et de résistance à l'usure. Les éléments du squelette du pneu, renforts métalliques ou textiles, sont associés par des matériaux élastomères, fortement déformables, aux propriétés adaptées aux différents fonctionnements thermomécaniques des matériaux du pneu en usage. Homogènes à des échelles supérieures au micron, ces matériaux sont, à plus petite échelle, des composites, faits de coupages de polymères, d'huiles, de charges renforçantes et d'additifs chimiques. Chaque matériau est un assemblage d'une dizaine de matières premières.

Pour des problèmes d'approvisionnement en caoutchouc naturel pendant la Seconde Guerre mondiale, la synthèse des polybutadiènes a connu un essor fulgurant. Des avancées majeures en terme d'adhérence des pneumatiques ont été réalisées via le développement des copolymères styrène-butadiène, polymères dont la température de transition vitreuse est ajustable par le taux de styrène dans la chaîne.

Ces gains n'auraient pu être valorisés dans l'industrie du pneu sans un renforcement des propriétés mécaniques de ces polymères par l'insertion d'agglomérats de particules minérales, appelés " charges ». L'apport du renforcement des polymères par les charges que sont le noir de carbone et la silice est double : le matériau chargé possède un module élastique typiquement 2 à 5 fois plus élevé que le polymère seul, et la contrainte à rupture d'un matériau chargé est multipliée par un facteur supérieur à 10. La contrepartie de ce renforcement est une augmentation des pertes hystérétiques dans le matériau lors de la déformation induite par le roulage du pneu, qui se traduit par une résistance à l'avancement. Le renforcement par la silice n'est effectif qu'en utilisant des polymères fonctionnels, ou des agents de couplage qui se lient par une extrémité à la charge et par l'autre au polymère.
Dans cet article, nous allons détailler les relations entre propriétés macroscopiques et structure microscopique du matériau, en intégrant l'interaction entre matières premières et procédé et l'apport de la simulation numérique.

\section{Procédé d'obtention et processus physico-chimiques}

Le processus de fabrication des gommes peut paraitre des plus rudimentaires : les polymères en morceaux et les charges sous forme de granulés de taille millimétrique sont introduits dans un mélangeur et malaxés, typiquement pendant une durée de 2 à 5 minutes. Le fait de cisailler des polymères très visqueux - la masse molaire moyenne d'un polymère de synthèse variant typiquement entre 50000 et $150000 \mathrm{~g} / \mathrm{mol}$ engendre une production de chaleur importante et donc une élévation de température, dépendant des conditions d'échange entre le mélangeur et son milieu extérieur. Un mélangeur industriel de $300 \mathrm{~kg}$ fonctionne en condition quasi adiabatique, et sa température interne atteint 160 à $170^{\circ} \mathrm{C}$ en quelques minutes. Associant température élevée et cisaillement mécanique, le mélangeur est en fait un réacteur chimique, et un examen détaillé des processus physico-chimiques mis en jeu révèle toute la complexité du procédé de synthèse des matériaux.

1 Fragmentation des agglomérats de charges : les charges introduites dans le mélangeur sont sous la forme d'agglomérats d'objets élémentaires insécables, appelés " agrégats ", dont on détaillera la structure ultérieurement. L'action première du mélangeur consiste à fragmenter les objets macroscopiques dans le but d'obtenir des agrégats individualisés en imposant un fort cisaillement de la matière. Un but second est de disperser et d'homogénéiser ces agrégats dans le polymère.

2 Fragmentation des chaînes de polymères : à haute température et sous forte extension lors des cisaillements, les chaînes de polymères sont susceptibles de se rompre. Cette fragmentation dépend essentiellement de la nature chimique de la chaîne de polymère. Dans le 
cas de polymères de synthèse, on pourra introduire des fonctions le long de la chaîne en quantité contrôlée et avec une chimie spécifique, permettant d'imposer la fragmentation à des températures variables.

3- Greffage sur la surface accessible des charges : plusieurs espèces sont aptes à se greffer sur les charges au cours du mélange. La chimie du « pied » des agents de couplage ou de la fonctionnalité d'un polymère fonctionnel a été spécifiquement conçue pour former un ancrage sur la charge. Dans le cas où la fragmentation du polymère est aussi un processus important, en particulier à haute température, les fragments peuvent former des liaisons avec la surface des charges. L'efficacité du greffage dépend fortement de l'accessibilité de la surface et des sites actifs, en particulier les surfaces vierges exposées suite à un événement de fragmentation des agglomérats.

La quantité de polymère greffé est mesurable, en réalisant une extraction du polymère non greffé par un bon solvant et une simple pesée après séchage. Cette quantification globale de polymère lié, dénommée Bound Rubber (BR) et exprimée en fraction de la quantité de polymère constitutif, varie fortement en fonction des matières premières utilisées et du temps de mélange.

\section{Les propriétés physiques et mécaniques des mélanges}

Une des propriétés mécaniques remarquable des polymères renforcés est l'effet Payne, caractérisé par une évolution de la rigidité du matériau avec l'amplitude de la déformation appliquée (fig. 1). On appelle non-linéarité (NL) de rigidité, la différence entre les modules élastiques à basse et grande déformation, NL $=G^{\star}(0,1 \%)-$ $\mathrm{G}^{\star}(100 \%)$, mesurés dans un essai de cisaillement simple à haute température. Le module $G^{\star}$ relie la contrainte $\sigma$ à la déformation de cisaillement sinusoïdale $\gamma$ appliquée via l'expression $\sigma=\mathrm{G}^{\star} \mathrm{e}^{\mathrm{i} \delta} \gamma$ avec $\gamma=\gamma_{0} \mathrm{e}^{\mathrm{i} \omega \mathrm{t}}$. L'effet Payne se traduit par une dissipation d'énergie d'origine viscoélastique reliée à l'angle de déphasage $\delta$ dans l'expression ci-dessus. Si la non-linéarité de rigidité n'est pas directement reliée à une performance du pneumatique, la dissipation d'énergie dans les mélanges impacte fortement la résistance au roulement liée au pneu. Cette résistance représente $20 \%$ de la

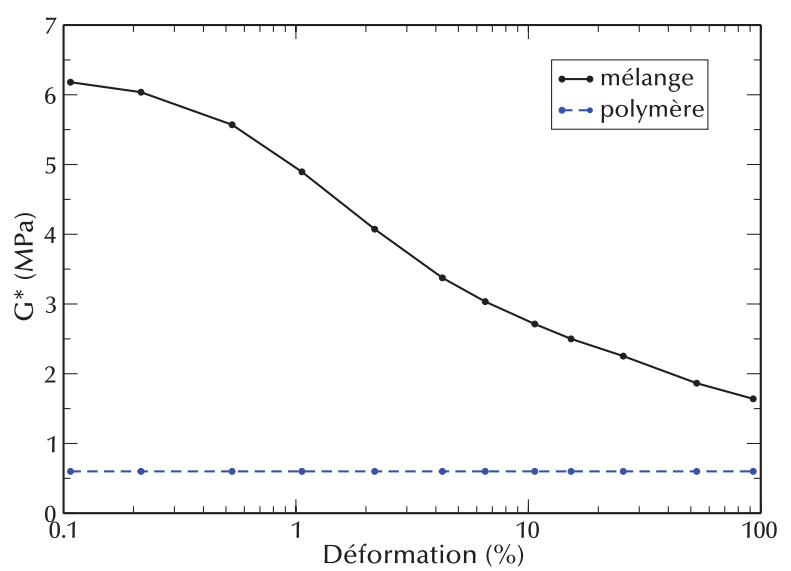

1. Module d'élasticité d'un mélange à base de noir de carbone N234 et du polymère constitutif en fonction de la déformation appliquée à l'éprouvette. Caractérisation réalisée en cisaillement simple à $60^{\circ} \mathrm{C}$ à $10 \mathrm{~Hz}$. On remarquera l'absence d'effet Payne dans le polymère pur.

consommation de carburant d'un véhicule de tourisme.

L'origine microscopique de la nonlinéarité, présente dans les mélanges pour tout type de charge renforçante, est un sujet de débat dans la communauté scientifique [1]. Parmi les mécanismes invoqués, on citera la rupture d'un réseau d'agrégats formé par floculation après mélange, la désorption/ adsorption de chaines de polymères sur les agrégats, ou la fusion d'une zone vitreuse de polymère à l'interface polymère/charge sous l'effet de la sollicitation mécanique. Dans le cas du noir de carbone, la mesure de la résistivité $\rho$ d'un mélange a été largement utilisée comme caractéristique " microscopique " de la structure des matériaux. La conduction électrique se fait entre les agrégats conducteurs d'électricité par effet tunnel et sonde ainsi les courtes distances entre les charges. Des travaux intensifs de nature "relations structurepropriétés » ont permis d'établir des corrélations (fig. 2) entre ces trois grandeurs caractéristiques : NL, BR et $\rho$. La corrélation entre NL et $\rho$ est interprétée par une origine commune de ces deux propriétés, toutes deux reliées à l'existence de courtes distances entre les charges ; la corrélation entre BR et $\rho$ comme une relation de cause à effet :
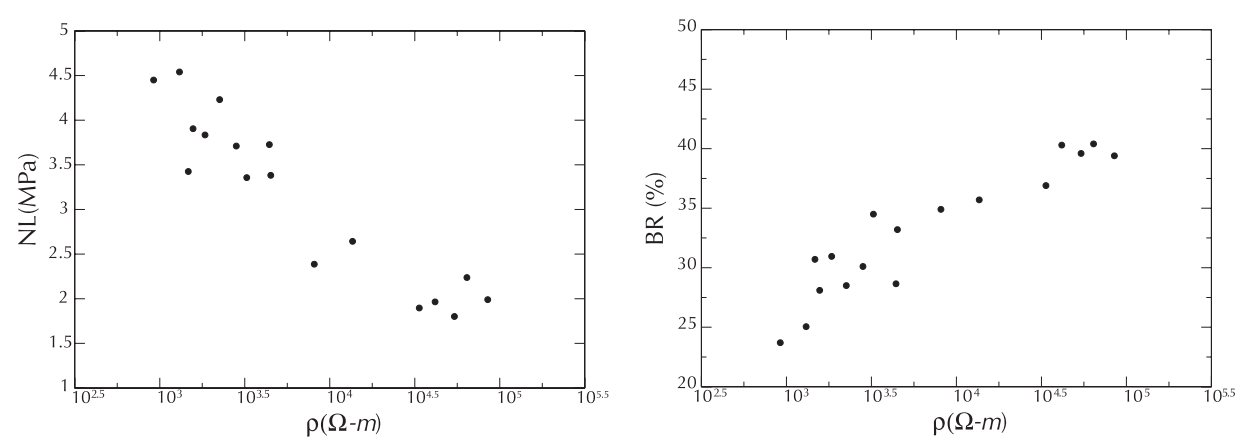

2. Corrélation entre amplitude de la non-linéarité et résistivité (a), et entre Bound Rubber et résistivité (b) de divers mélanges à base de différents polymères (naturels ou synthétiques) avec un noir de carbone N234 dans diverses conditions de mélangeage.

plus la quantité de polymère greffé est grande et plus les distances entre les charges sont grandes et le mélange résistif.

Comme en témoignent les graphes de la figure 2 , les tendances restent qualitatives et de nombreux travaux sont nécessaires pour passer à une description quantitative du rôle du polymère greffé sur l'établissement des distances entre les agrégats et le lien avec l'effet Payne.

\section{Les agrégats de charge}

On a évoqué précédemment le processus de fragmentation des agglomérats dans le mélangeur : ce processus doit être le plus abouti possible pour tirer le meilleur parti de la matière première en dissociant un maximum d'agglomérats en agrégats élémentaires. La caractérisation de ces agrégats élémentaires supposés insécables est réalisée en couplant plusieurs techniques expérimentales. En particulier l'imagerie directe par microscopie électronique en transmission de haute résolution permet de déterminer les caractéristiques fondamentales des agrégats (fig. 3) : ceux-ci sont composés de particules élémentaires, sphériques en première approximation, ayant une distribution de rayon s'étalant entre 10 et $30 \mathrm{~nm}$

$>>>$ . 


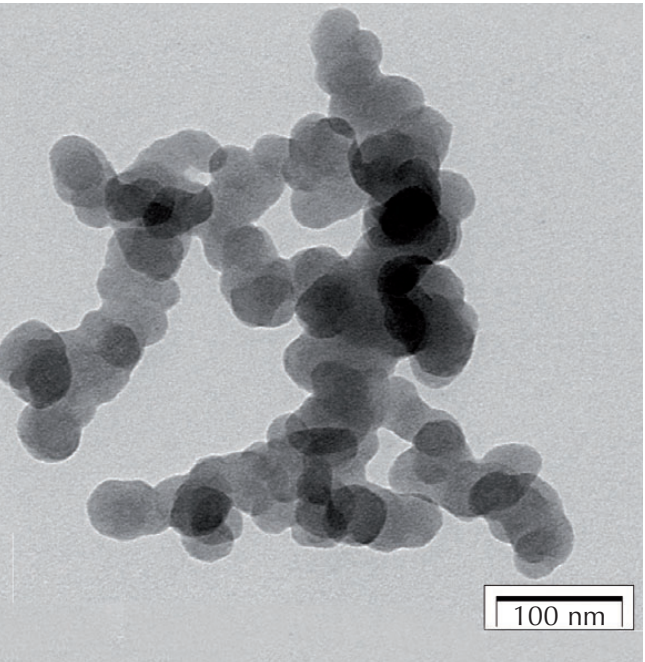

3. Image de microscopie électronique en transmission d'un agrégat de noir de carbone $(1$ pixel $=0.5 \mathrm{~nm})$. Les agglomérats sont traités dans l'eau avec une sonde à ultrasons qui permet la fragmentation totale. Une goutte de liquide est déposée sur une grille de microscopie et le solvant est évaporé. L'image étant une projection 2D d'un objet tridimensionnel, 3 niveaux de gris se distinguent : gris clair, gris foncé et noir, quand le faisceau électronique traverse respectivement 1, 2 ou plus de 3 particules élémentaires.

$>>>$

pour un noir de carbone. Lors de la synthèse des charges, des particules élémentaires en croissance vont entrer en collision et fusionner de manière irréversible selon différents processus : processus balistique dans le cas des milieux très dilués qui est celui des phases gazeuses à très hautes températures, processus brownien dans le cas des milieux concentrés ou en phase liquide. Ces différents types de processus conduisent à la formation d'objets fractals, plus ou moins dendritiques ou compacts [2]. Il existe une distribution du nombre de particules par agrégats allant de 2 à plus de 100 particules élémentaires, leur sphère circonscrite ayant un rayon pouvant atteindre 200 à $300 \mathrm{~nm}$.

\section{Les deux phases du procédé de fabrication}

Pour revenir aux mécanismes physiques mis en jeu dans le procédé de synthèse des matériaux, on va considérer qu'il existe deux phases bien distinctes qui permettent de faire des hypothèses simplificatrices utiles pour la simulation numérique.

1 Une première phase, pendant laquelle l'action du rotor du mélangeur entraîne une décorrélation rapide de la position des charges dans le mélange. Cette phase peut être assimilée à l'obtention d'une répartition aléatoire d'agrégats élémentaires, si le processus de fragmentation a atteint son stade ultime, ou plus généralement d'objets présents au temps t écoulé depuis le début du processus de fragmentation.

4. Image de microscopie électronique à balayage reconstruite par simulation en donnant aux charges le contraste d'objets de silice dans une matrice de polymère diénique $(1$ pixel = 2,1 nm). La fraction volumique de charge est de $18 \%$. Chaque particule élémentaire d'un agrégat est représentée par un disque blanc dans le plan de coupe et dans un niveau de gris croissant avec la profondeur jusqu'à une distance de $10 \mathrm{~nm}$ de la coupe. 0n remarquera que l'image de cette coupe de matériau contraste avec la quasi-percolation 3D de la structure, obtenue via un nombre de contacts faible par rapport au nombre de particules dans le système.
Toute observation à la fin de la première étape reste un challenge pour l'expérimentateur. En revanche, il faut garder à l'esprit que le mélange final sur lequel on peut réaliser des mesures est la résultante de ces deux processus faisant appel à des physiques très différentes.

\section{Analyse des répartitions aléatoires par simulation}

Pour être représentatif d'un échantillon macroscopiquement homogène, il est nécessaire de travailler sur des échantillons de 1 à 2 microns de côté. La fraction volumique cible étant comprise entre 15 et $25 \%$ de charges, cela nécessite de construire quelques milliers d'agrégats à partir de quelques centaines de milliers de particules élémentaires respectant la dimension fractale du processus d'agrégation et les distributions en rayon de particules et en nombre de particules décrites ci-dessus. Cette tâche réalisée, on utilise un algorithme élémentaire de répartition aléatoire des agrégats dans une boîte cubique. Le succès de la méthode tient au fait que les fractions volumiques cibles sont largement inférieures à la compacité maximale des agrégats, estimée entre 35 et $40 \%$. On s'est intéressé dans un premier temps à analyser par simulation les dispersions aléatoires d'agrégats, en se focalisant sur le calcul des courtes distances entre les agrégats dans le cas d'une fragmentation totale.

Une vision de la topologie du milieu est donnée en calculant le nombre d'objets distincts et le nombre de particules dans le plus gros objet en fonction d'une distance caractéristique $d$. Pour ce faire, on se donne la règle suivante : deux agrégats appartiennent au même objet si les particules les plus proches sont séparées par une distance inférieure à $\mathrm{d}$, qui peut être assimilée à la taille d'un pixel d'une image de microscopie (fig. 4). Le nombre de particules dans le plus gros objet présente une transition très abrupte pour une distance de quelques nanomètres, à laquelle on atteint un seuil de

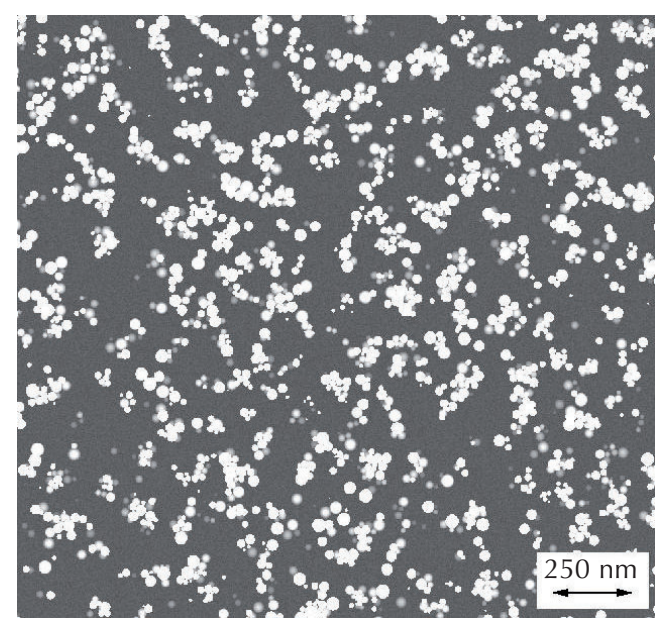




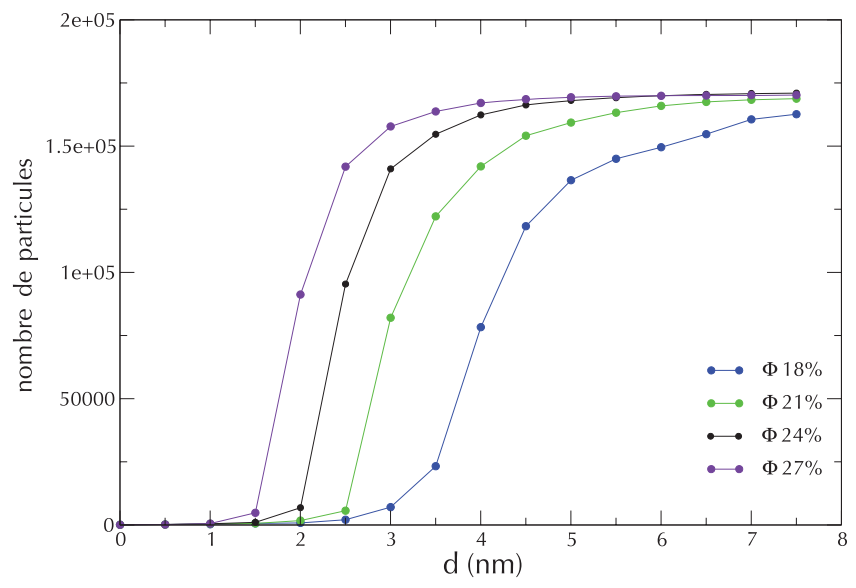

5. Nombre de particules dans le plus gros objet en fonction d'une distance de distinction $\mathbf{d}$ : deux agrégats appartiennent au même objet si la distance minimale entre les particules constitutives est inférieure à d. Le système est constitué de 7200 agrégats élémentaires, construits avec 170000 particules environ. Une fraction volumique de 18\% représente un cube de matière de 2 um d’arête.

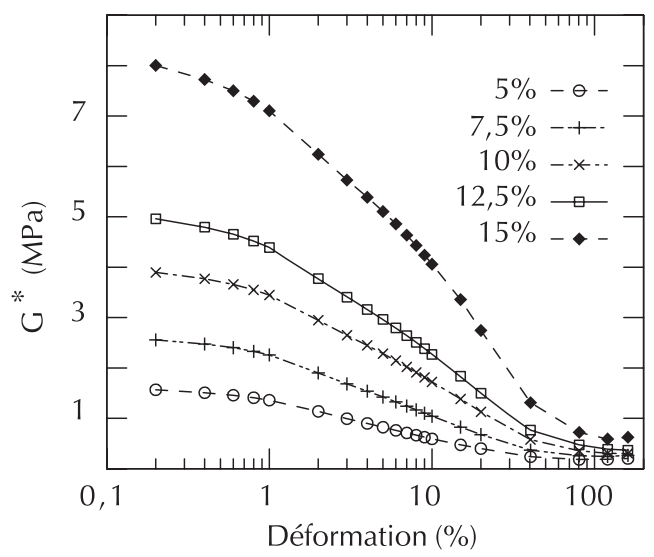

6. Module d'élasticité de mélanges obtenus par simulation numérique pour différentes fractions volumiques de charge. Le modèle est construit avec les seuls éléments suivants : un potentiel quadratique assimilable à un ressort est imposé entre les particules d'agrégats différents les plus proches dans l'état initial ; cette connectique, traduisant l'interaction via le polymère confiné reste inchangée durant la déformation. Un potentiel à courte portée de type Lennard-Jones entre toutes les particules est ajouté pour interdire l'interpénétration entre particules rigides lors de la déformation.

\section{Réérences}

1 - T.A. Witten, M. Rubinstein et R. H. Colby, "Reinforcement of Rubber by Fractal Aggregates", J. Phys. // 3 (1993) 367-383 ; M. Klüppel, "The Role of Disorder in Filler Reinforcement of Elastomers on Various Length Scales", Adv. Polym. Sci. 161 (2003) 1-86 ; S. Merabia, P. Sotta et D. Long, "A Microscopic Model for the Reinforcement and the Nonlinear Behavior of Filled Elastomers and Thermoplastic Elastomers (Payne and Mullins Effects)", Macromolecules 41 (2008) 8252-8266.

2 • Aggregation and Fractal Aggregates, R. Jullien et R. Botet, World Scientific (1987).

3 - K. Smith, M. Vladkov et J.L. Barrat, “Polymer Melt near a Solid Surface: A Molecular Dynamics Study of Chain Conformations and Desorption Dynamics", Macromolecules 38 (2005) 571-580 ; M. Vladkov et J.L. Barrat, "Local Dynamics and Primitive Path Analysis for a Model Polymer Melt near a Surface", Macromolecules 40 (2007) 3797-3804.

4 J.D. Thomin, P. Keblinski et S.K. Kumar, "Network Effects on the Nonlinear Rheology of Polymer Nanocomposites", Macromolecules 41 (2008) 5988-5991.

$5 \bullet$ D. Long et P. Sotta, "Nonlinear and Plastic Behavior of Soft Thermoplastic and Filled Elastomers studied by Dissipative Particle Dynamics", Macromolecules 39 (2006) 6282-6297. percolation des agrégats de charges qui forment alors un amas couvrant tout l'échantillon (fig. 5). En augmentant la fraction volumique de charge de 18 à $27 \%$, on obtient la transition percolante vers des distances de distinction de plus en plus faibles. La morphologie dendritique des agrégats et la répartition aléatoire sont à l'origine des distances de quelques nanomètres entre ceux-ci.

Les propriétés macroscopiques des mélanges sont la résultante d'une cascade d'effets physiques à différentes échelles. Ainsi, la répartition aléatoire des charges, prise comme état initial de l'auto-organisation avant auto-assemblage sous l'effet des forces interparticulaires, est caractérisée par des distances de l'ordre de quelques nanomètres. Cette distance est du même ordre de grandeur que le rayon de giration d'une chaîne de polymère dans un fondu libre. Le polymère dans le mélange se trouve donc confiné entre les surfaces des charges et son interaction avec les sites surfaciques modifie ses caractéristiques morphologiques et sa dynamique par rapport au fondu libre [3]. Une chaîne de polymère a la faculté de pouvoir interagir avec deux particules de charge éloignées de quelques nanomètres. Il a été montré par simulation numérique que ce phénomène local engendrait des effets de réseaux macroscopiques à l'origine de l'existence d'un pic de contrainte en début d'écoulement [4].

On a aussi cherché à analyser par simulation numérique la contribution à l'effet Payne des deux spécificités majeures : la morphologie des agrégats et l'interaction spécifique entre les particules séparées par quelques nanomètres via des chaînes de polymère. Dans ce dessein, on a étendu un modèle développé précédemment pour des particules sphériques [5] aux agrégats supposés indéformables et mobiles, sous l'effet des différentes forces, par translation du centre de masse et rotation dans un milieu de viscosité constante. Les résultats de simulation (fig. 6) montrent une forte non-linéarité du module. L'interaction spécifique entre les particules proches est un élément clé de l'effet Payne et son origine physique aujourd'hui controversée reste à établir.

\section{Conclusion et perspectives}

La simulation numérique de répartitions aléatoires d'agrégats de charge dans une matrice de polymère a permis de mettre en évidence des valeurs de distances interagrégats de l'ordre de quelques nanomètres. En postulant l'existence d'une interaction spécifique à courte distance entre polymère et charge, la simulation numérique des propriétés mécaniques retrouve l'effet Payne caractéristique de ces matériaux. Cette interaction à l'échelle microscopique a des répercussions à l'échelle macroscopique sur les propriétés mécaniques et, par conséquent, sur la performance des pneus et la consommation de carburant des véhicules. Élucider son origine physique est un point fondamental. L'objectif final de la démarche est de construire une simulation numérique permettant de prédire quantitativement les propriétés macroscopiques et permettre l'optimisation du couple matières premières - procédé pour accroître la puissance de l'innovation matériau.

Remerciements

Cet article est extrait d'une présentation au minicolloque "Particules colloïdales et nano-objets : auto-organisation et inclusions dans les fluides complexes " des Journées de la Matière Condensée 2008. Je tiens à remercier les organisateurs pour l'invitation à présenter ces travaux. 